\title{
Oscilación de un disco giratorio visto desde un sistema de referencia en rotación
}

\author{
Oscillation of a spinning disc viewed from a rotating frame
}

\author{
D A Martínez ${ }^{1}$, A Rela ${ }^{2}$, J Sztrajman*3 \\ ${ }^{1}$ Universidad Nacional de Río Negro, Río Negro, Argentina \\ ${ }^{2}$ Instituto Municipal de Educación Superior de Formación Docente Capacyt, Buenos Aires, Argentina \\ ${ }^{3}$ Universidad de Buenos Aires, Buenos Aires, Argentina
}

Received on October 21, 2016. Revised on December 09, 2016. Accepted on December 16, 2016.

Con un modelo de juguete, se calcula la relación 1:2 entre las frecuencias de rotación y de oscilación de un disco giratorio libre de torques, tal como se lo ve desde un sistema de referencia inercial. Desde un conveniente sistema de referencia en rotación, el cuerpo oscila como un péndulo en un campo centrífugo, lo que simplifica la descripción de su movimiento. La relación de frecuencias es más simple de obtener cuando el movimiento se analiza primeramente desde un sistema de referencia en rotación, y después desde uno inercial. Con este método se deduce también la relación de frecuencias para un disco plano. El enfoque parece útil en la enseñanza de la mecánica elemental a estudiantes que aún no se graduaron, porque evita dificultades que surgen cuando el problema se resuelve empleando métodos de la mecánica analítica.

Palabras clave: mecánica clásica, disco giratorio, sistema de referencia en rotación.

The ratio relation 1:2 between rotation and wobbling frequencies of a torque free spinning plate or disc as seen from an inertial frame is calculated using a toy model for the disc. From a convenient rotating frame, the body oscillates as a pendulum in a centrifugal field, which simplifies the description of its movement. Thus, the ratio relation is simpler obtained when the system is first analyzed from a rotating frame and then from an inertial one. Using this approach, the derivation of the ratio relation for a flat disc is shown too. This appears useful when teaching elementary mechanics to undergraduate students because it avoids the difficulties that appear when the problem is solved using methods of analytical mechanics.

Keywords: classical mechanics, spinning disc, rotating frame.

\section{Introducción}

El movimiento de un cuerpo rígido libre de torques es ciertamente complejo. Un caso muy bien estudiado es, por ejemplo, el de la rotación inercial de un cuerpo rígido. Recientemente, se han puesto de manifiesto las dificultades en la comprensión del mismo de los estudiantes de grado $[1,2]$. Con el objeto de contribuir a superar esas dificultades, mostramos aquí una forma de facilitar su comprensión en el caso particular de un disco giratorio analizado desde un sistema de referencia rotante.

*Endereço de correspondência: jsztraj@gmail.com
Las anécdotas más interesantes de Richard Feynman son aquellas que ilustran su entusiasmo por explicar cómo funcionan las cosas independientemente de su importancia.

Sólo por diversión. Una de ellas es la que relata cómo halló el camino para la formulación de su versión de la electrodinámica cuántica a partir de la observación de un fenómeno aparentemente sin mucha importancia: la relación entre las frecuencias de rotación y de oscilación de una bandeja rotante [3] en ausencia de torques. En sus propias palabras [4]:

Mientras la bandeja volaba dando vueltas, me fijé en que había en ella un me- 
dallón de Cornell. La bandeja giraba y se bamboleaba, y saltaba a la vista que el medallón giraba más rápidamente de lo que se bamboleaba. No tenía nada que hacer, así que me puse a calcular cuál sería el movimiento de la bandeja giratoria. Descubrí que cuando el ángulo es muy pequeño, la velocidad del giro del medallón es doble del ritmo de bamboleo. Una relación de 2 a 1 . Así se deducía de una complicada ecuación. Entonces pensé: "¿No habrá forma de verlo desde un enfoque más fundamental, analizando las fuerzas, o la dinámica del movimiento, para ver por qué la relación ha de ser de 2 a 1 ?".

La relación correcta es 1:2, no 2:1. Varios autores notaron este error [5], pero es irrelevante comparado con el interés por el hecho descrito por Feynman.

La relación correcta puede obtenerse empleando las ecuaciones de Euler para un disco rotante [3].

Varios autores han propuesto demostraciones alternativas que ayudan a la comprensión del fenómeno, como el empleo de un modelo de juguete (toy model) con dos partículas sujetas a barras de masa despreciable y formando un ángulo de $90^{\circ}$ entre ellas [6], o basados en un análisis de las fuerzas que actúan sobre cada partícula del disco desde un sistema fijo a Tierra (inercial) [7].

Mostramos aquí una forma alternativa de obtener el mismo resultado también con un modelo de juguete sin emplear ecuaciones complicadas, analizando primero el movimiento desde un sistema rotante (sección 2) y, posteriormente, usando un resultado obtenido del análisis desde este sistema, desde un sistema de referencia inercial (sección 3).

En la sección 4 se describe como obtener la relación 1:2 para un disco rotante, empleando la misma técnica.

\section{El modelo de juguete desde un sistema rotante}

El modelo consiste en una cruz formada por cuatro pequeñas esferas de masa $m$, ligadas a dos barras de masa despreciable, cada una de longitud $2 a$.

Consideremos que la cruz rota con una velocidad angular $\omega$ alrededor de un eje perpendicular al plano que contiene las cuatro esferas (eje $Z$ ). Desde un sistema rotante que gira con la misma velocidad angular $\Omega=\omega$ las fuerzas centrífugas no producen ningún efecto neto, y la cruz permanece en reposo (Fig. 1).

Si en el instante $t=0$ las esferas ubicadas sobre el eje Y se empujan ligeramente con una velocidad $\mathrm{v}$, el eje del disco experimenta un apartamiento pequeño y el sistema comienza a oscilar con una frecuencia angular $\omega_{0}=2 \pi / T$, donde $T$ es el período de oscilación (Fig. 2).

Es importante notar que para pequeños apartamientos de la posición de equilibrio las fuerzas de Coriolis pueden despreciarse, pues la velocidad $\mathbf{v}$ de las esferas es casi paralela al vector velocidad angular $\Omega$ del sistema rotante.

La frecuencia de oscilación puede calcularse fácilmente teniendo en cuenta que, al igual que la fuerza gravitatoria $m g$ que actúa sobre la lenteja de un péndulo, la fuerza centrífuga $m a \Omega^{2} \cos \theta$ sobre cada esfera actúa como fuerza restauradora, donde la aceleración centrífuga juega el rol de g (Fig. 3). Para ángulos pequeños $(\theta<<1, \cos \theta \cong 1)$, la fuerza centrífuga puede aproximarse por $m a \Omega^{2}$.

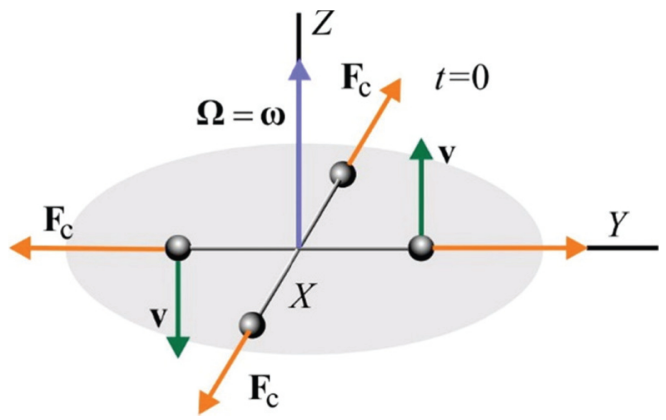

Figura 1: Una cruz rotando alrededor del eje $Z$, se observa en reposo desde un sistema rotante con la misma velocidad angular. En el instante $t=0$, las esferas ubicadas sobre el eje $\mathrm{Y}$ se empujan ligeramente con una velocidad $\mathbf{v}$. $\mathbf{F}_{\mathbf{c}}$ : fuerza centrífuga.

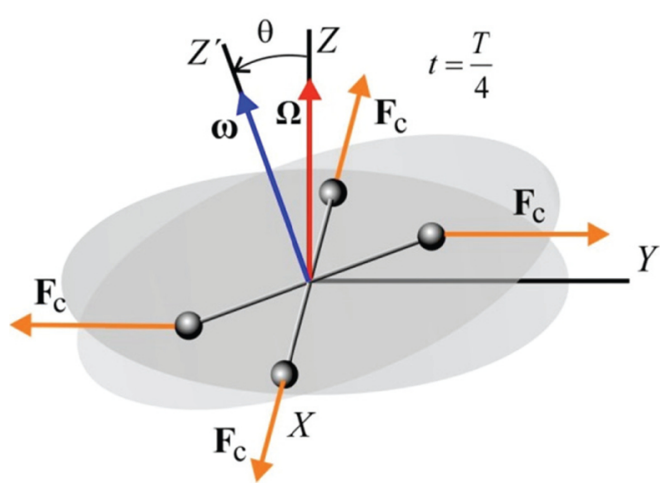

Figura 2: En $t=T / 4$ se alcanza un apartamiento máximo $\theta$ del eje $Z$ y las fuerzas centrífugas tienen componentes perpendiculares a las barras. 


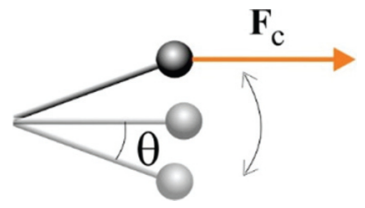

Figura 3: El sistema oscila como un péndulo en un campo de fuerza centrífugo.

Como la frecuencia de oscilación de un péndulo de longitud $a$ está dada por la conocida expresión $\sqrt{g / a}$, la frecuencia de oscilación de la cruz resulta:

$$
\omega_{o}=\sqrt{\frac{\Omega^{2} a}{a}}=\Omega
$$

De esta forma se obtiene que, vistas desde el sistema rotante, las esferas inicialmente localizadas sobre el eje $Y$ oscilan en el plano $Y Z$ con una frecuencia angular igual a la velocidad angular de la cruz, mientras que las esferas ubicadas sobre el eje $X$ permanecen en reposo.

\section{El modelo de juguete analizado desde un sistema inercial}

Se demostró anteriormente que, desde un sistema rotante, la frecuencia de oscilación de la cruz coincide con la velocidad angular del sistema de referencia. Desde un sistema de referencia inercial (no rotante) se observa algo distinto.

Si se identifican las esferas con los números 1, 2 , 3 y 4 , como muestra la Fig. 4, en el instante $t=0$ la esfera 2 se mueve hacia arriba, mientras que la 4 lo hace hacia abajo.

Un cuarto de período más tarde, la esfera 4 yace en el plano $X Z$ y por debajo del plano $X Y$ (Fig. 5)

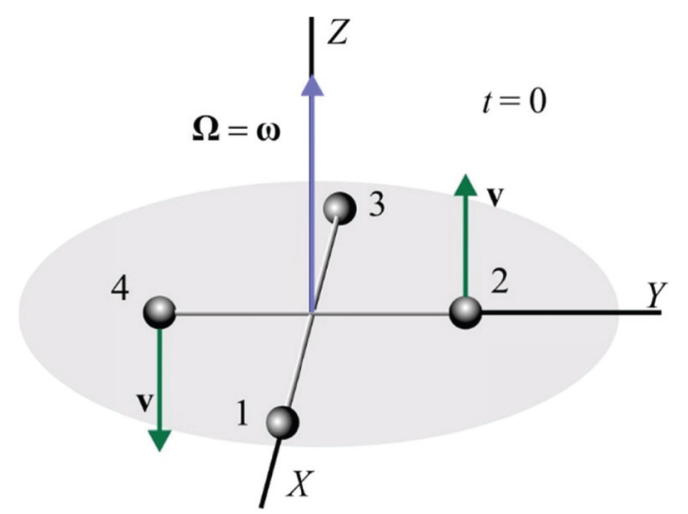

Figura 4: Desde un sistema de referencia inercial, en $t=$ 0 la esfera 2 se mueve hacia arriba y la 4 hacia abajo.

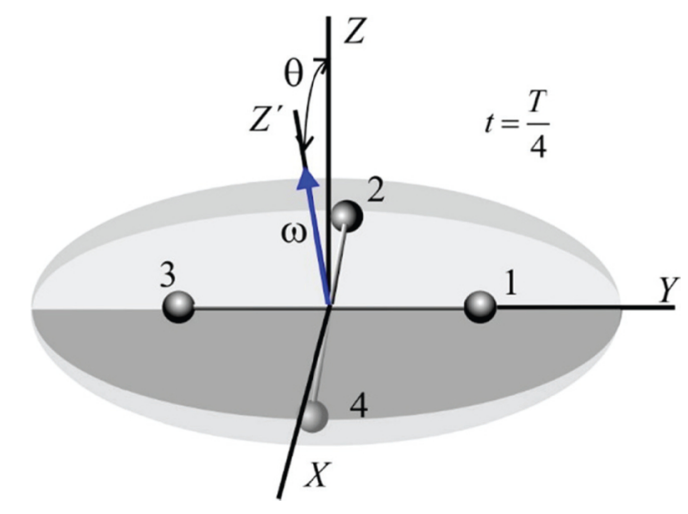

Figura 5: Un cuarto de período más tarde, las esferas 2 y 4 se encuentran sobre el plano $X Y$, con la esfera 4 por debajo del mismo y la 2 por encima.

-recordemos que el período de oscilación es igual al período de rotación-.

Como la esfera 3 no se está moviendo ni hacia arriba ni hacia abajo, en el instante $t=T / 2$ se hallará sobre el eje $X$ (Fig. 6).

En este instante $(T / 2)$, la esfera 2 se está moviendo hacia abajo, de modo que se ubicará por debajo del plano $X Y$ un cuarto de período más tarde (Fig. 7).

Si cada cuarto de período se registra la posición de la esfera ubicada en el plano $X Z$ y dirigida hacia el lector $($ eje $+X)$, independientemente de su número, se verá que ésta experimenta dos oscilaciones durante una vuelta completa de la cruz (cada esfera identificada con un número oscila una vez por cada vuelta). Por lo tanto, la relación entre las frecuencias de rotación y oscilación de una cruz formada por cuatro esferas unidas por barras de masa despreciable y en ausencia de torques es 1:2.

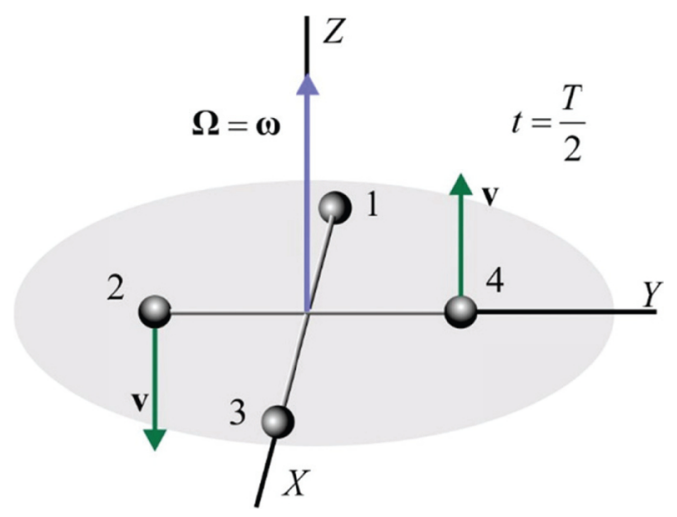

Figura 6: En el instante $T / 2$ la esfera 2 se mueve hacia abajo y la 4 hacia arriba. 


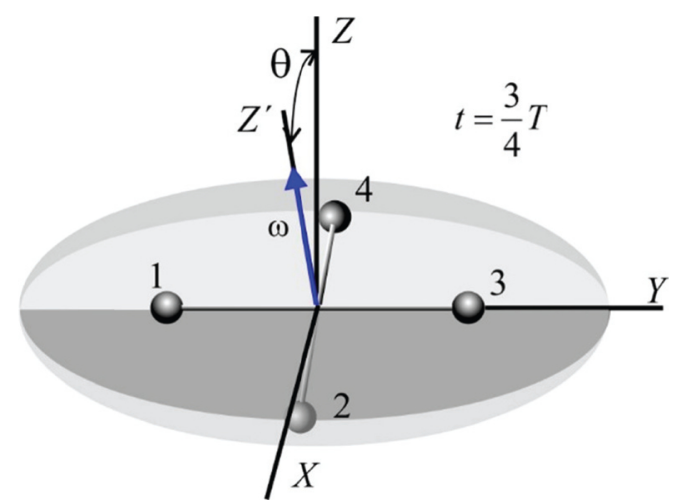

Figura 7: Al tiempo $3 T / 4$, la esfera 2 se encuentra por debajo y la 4 por encima de $X Y$.

\section{El disco}

Hasta aquí, hemos considerado un modelo de juguete que ayuda a comprender la relación entre las frecuencias de rotación y oscilación sin hacer uso de matemática avanzada. Consideremos ahora el modelo más real de una bandeja que consiste en un disco plano homogéneo de radio a y masa $M$, rotando con una velocidad angular $\omega$ según una dirección perpendicular al plano que lo contiene.

Analizado desde un sistema rotante con la misma velocidad angular, éste se verá en reposo. Sin embargo, si el disco se tuerce ligeramente respecto de su eje $X$, alcanzará una orientación tal como muestra la Fig. 8.

Sobre cada elemento de masa diferencial $\mathrm{d} m$

$$
\mathrm{d} m=\frac{M}{\pi a^{2}} r \mathrm{~d} r \mathrm{~d} \varphi
$$

cuya posición está dada por las coordenadas polares $r$ and $\varphi$, actúa una fuerza centrífuga $\mathrm{d} \mathbf{F}_{\mathbf{c}}$ dirigida en forma perpendicular al eje $Z$, como muestra la Fig. 9.

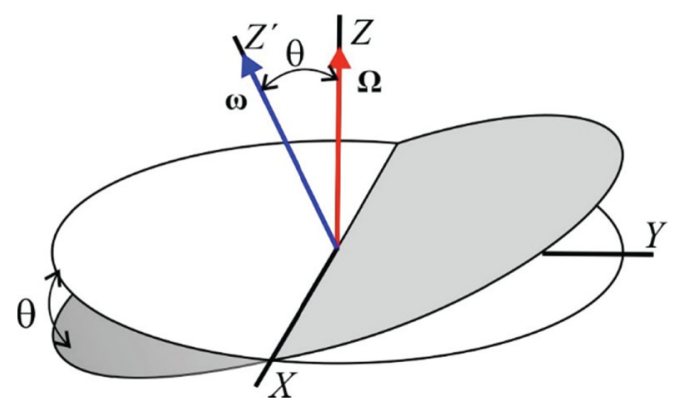

Figura 8: Visto desde un sistema de referencia rotante, un disco plano giratorio que se tuerce ligeramente respecto de su eje $X$ adquirirá una orientación con su eje $Z^{\prime}$ apartado un ángulo máximo $\theta$.

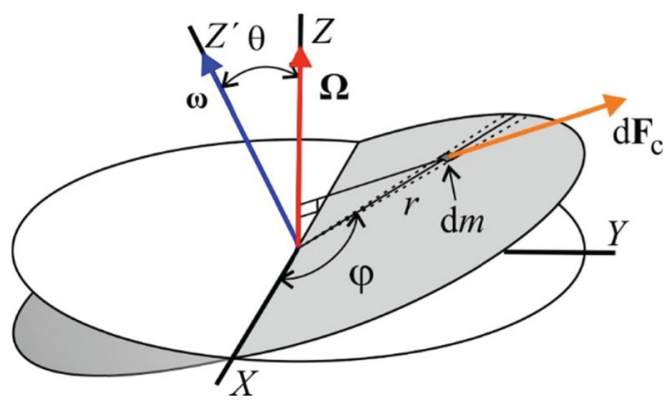

Figura 9: Un elemento de masa $\mathrm{d} m$ con coordenadas ry $\phi$ sobre el disco experimentará una fuerza centrífuga $\mathrm{d} F_{c}$ perpendicular al eje $Z$.

La intensidad de esta fuerza es:

$$
\mathrm{d} F_{c}=\mathrm{d} m \omega^{2} r \cos \theta=\frac{M}{\pi a^{2}} \omega^{2} r^{2} \cos \theta \mathrm{d} r \mathrm{~d} \varphi
$$

que para pequeños desplazamientos angulares $(\theta<<$ $1, \cos \theta \cong 1$ ) puede expresarse como:

$$
\mathrm{d} F_{c}=\frac{M}{\pi a^{2}} \Omega^{2} r^{2} \mathrm{~d} r \mathrm{~d} \varphi
$$

Las componentes $X$ e $Y$ de este elemento de fuerza son:

$$
\mathrm{d} F_{c x}=\frac{M}{\pi a^{2}} \Omega^{2} r^{2} \cos ^{2} \varphi \mathrm{d} r \mathrm{~d} \varphi
$$

y:

$$
\mathrm{d} F_{c y}=\frac{M}{\pi a^{2}} \Omega^{2} r^{2} \sin ^{2} \varphi \mathrm{d} r \mathrm{~d} \varphi
$$

respectivamente.

Al sumar (integrar) todas las componentes $X$ de las fuerzas, éstas se anulan por razones de simetría. De modo que la componente de interés es $\mathrm{d} F_{c y}$.

Esta componente produce un torque elemental $\mathrm{d} \tau_{x}$ (Fig. 10) dado por:

$$
\mathrm{d} \tau_{x}=-\frac{M}{\pi a^{2}} \Omega^{2} r^{3} \theta \sin ^{2} \varphi \mathrm{d} r \mathrm{~d} \varphi
$$

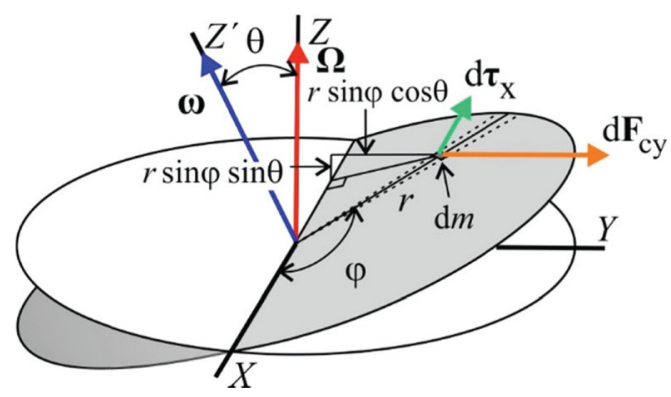

Figura 10: Componente $Y$ of $\mathrm{d} \boldsymbol{F}_{\mathbf{c}}$ y el torque $\mathrm{d} \tau_{\mathrm{x}}$ que produce. 
El torque total se obtiene integrando entre los límites $\varphi: 0 \rightarrow 2 \pi \quad r: 0 \rightarrow a:$

$$
\tau_{x}=-\frac{M}{\pi a^{2}} \Omega^{2} \theta\left(\int_{0}^{a} r^{3} \mathrm{~d} r\right)\left(\int_{0}^{2 \pi} \sin ^{2} \varphi \mathrm{d} \varphi\right)
$$

(El signo menos indica que el torque está dirigido según $-X)$.

Teniendo en cuenta que:

$$
\int_{0}^{2 \pi} \sin ^{2} \varphi \mathrm{d} \varphi=\pi
$$

de la ecuación (8) se obtiene que:

$$
\tau_{x}=-\frac{M a^{2}}{4} \Omega^{2} \theta
$$

Como el momento de inercia de un disco plano de radio a y masa $M$ respecto de un eje que pasa por su centro y sobre el plano del disco es:

$$
I=\frac{M a^{2}}{4}
$$

la ecuación (10) puede escribirse:

$$
\tau_{x}=-I \Omega^{2} \theta
$$

y el torque restaurador $\tau_{x}$ :

$$
\tau_{x}=I \frac{\mathrm{d}^{2} \theta}{\mathrm{d} t^{2}}
$$

De 12) y (13) se obtiene la conocida ecuación del oscilador armónico:

$$
\frac{\mathrm{d}^{2} \theta}{\mathrm{d} t^{2}}+\Omega^{2} \theta=0
$$

De modo que la frecuencia de oscilación del disco plano está dada también por la ecuación (1):

$$
\omega_{o}=\Omega
$$

Habiendo demostrado que, desde un sistema de referencia rotante, la frecuencia de oscilación del disco plano es igual a la obtenida para el modelo de juguete en forma de cruz, los argumentos utilizados en la sección 3 pueden ser fácilmente aplicados también aquí.

De los mismos se concluye que la relación entre las frecuencias de rotación y oscilación de un disco rotante en ausencia de torques es también 1:2.

En la Web pueden encontrarse simulaciones que permiten visualizar el fenómeno del bamboleo de un disco rotante $[2,7]$. Usando parte del código en lenguaje $\mathrm{C}$ escrito por Chris Hecker [8], hemos desarrollado dos programas que simulan en $3 \mathrm{D}$ la cruz $\mathrm{y}$ el disco girando, tanto vistos desde un sistema inercial como desde uno rotante. Los mismos se encuentran disponibles en https://www.mediafire com/folder/b0nxtr3j12s90/Animation.

\section{Conclusión}

En este trabajo hemos analizado la oscilación de un disco rotante libre de torques. Este movimiento presenta una relación interesante entre las frecuencias de rotación y de oscilación que pueden derivarse fácilmente usando un modelo de juguete, primeramente observado desde un sistema de referencia rotante y luego pasando a un sistema inercial. Esperamos que este método pueda ser de utilidad para la enseñanza de este movimiento complejo a estudiantes de pre-grado.

\section{Agradecimientos}

Agradecemos enormemente a Lorena Rela por la lectura cuidadosa del manuscrito y sus valiosos comentarios. También agradecemos al revisor anónimo de Ensino de Física por la sugerencia de incluir referencias bibliográficas adicionales sobre la misma temática desarrollada en este artículo.

Este trabajo fue parcialmente financiado por la Universidad de Buenos Aires, Programación Científica 2014-2017, UBACyT 20020130100390BA.

\section{Referencias}

[1] E. Butikov, Eur. J. Phys. 27, 913 (2006).

[2] S. Zabunov and M. Gaydarova, Lat. Am. J. Phys. Educ. 7, 192 (2013).

[3] J. Mehra, The Beat of a Different Drum. The Life and Science of Richard Feynman (Clarendon Press, Oxford, 1994) p. 173.

[4] R. Feynman, ¿Está Usted de Broma, Sr. Feynman?: Aventuras de un Curioso Personaje Tal Como Dueron Referidas a Ralph Leighton (Alianza Editorial, Madrid, 1997).

[5] B. Chao, Phys. Today 42, 15 (1989).

[6] A. Ruina, Phys. Today 42, 11 (1989).

[7] S. Tuleja, B. Gazovic, A. Tomori and J. Hanc, Am. J. Phys. 75, 240 (2007).

[8] C. Hecker, Rigid Body Dynamics, disponible en http://chrishecker.com/Rigid_Body_ Dynamics, accesado en 19/10/2016. 